\title{
Absence of a giant Rashba effect in the valence band of lead halide perovskites
}

\author{
M. Sajedi, ${ }^{1,2}$ M. Krivenkov, ${ }^{1,2}$ D. Marchenko, ${ }^{1}$ A. Varykhalov, ${ }^{1}$ J. Sánchez-Barriga, ${ }^{1}$ E. D. L. Rienks, ${ }^{1}$ O. Rader ${ }^{1}$ \\ 1 Helmholtz-Zentrum Berlin für Materialien und Energie, \\ Albert-Einstein-Str. 15, 12489 Berlin, Germany and \\ 2 Institut für Physik und Astronomie, Universität Potsdam, \\ Karl-Liebknecht-Str. 24/25, 14476 Potsdam, Germany
}

\begin{abstract}
For hybrid organic-inorganic as well as all-inorganic lead halide perovskites a Rashba effect has been invoked to explain the high efficiency in energy conversion by prohibiting direct recombination. Both a bulk and surface Rashba effect have been predicted. In the valence band of methylammonium (MA) lead bromide a Rashba effect has been reported by angle-resolved photoemission and circular dichroism with giant values of 7 to $11 \mathrm{eVA}$. We present band dispersion measurements of $\mathrm{MAPbBr}_{3}$ and spin-resolved photoemission of $\mathrm{CsPbBr}_{3}$ to show that a large Rashba effect detectable by photoemission or circular dichroism does not exist and cannot be the origin of the high effciency.
\end{abstract}

Since they merge outstanding optoelectronic characteristics such as tunable bandgap, high carrier mobility and long carrier lifetimes, lead halide perovskites (LHPs) have attracted outstanding attention for their application in multi-junction solar cells 1 resulting in high power conversion efficiencies exceeding $25 \%$ [2 6]. Due to high spin-orbit interaction, they have recently also been considered as material for spintronics $[7[-9]$. Metal halide perovskites possess an $A M X_{3}$ structure (where $A$ can be an organic cation such as methylammonium (MA), i. e., $\mathrm{CH}_{3} \mathrm{NH}_{3}^{+}$, or an inorganic cation such as $\left.\mathrm{Cs}^{+}\right), M$ is a metal $\left(\mathrm{Pb}^{2+}\right.$ but also $\left.\mathrm{Sn}^{2+}\right)$ and $X$ is a halide anion $\left(\mathrm{I}^{-}\right.$, $\mathrm{Br}^{-}$, or $\left.\mathrm{Cl}^{-}\right)$. $\mathrm{MAPbBr}_{3}$ undergoes a phase transition from cubic to tetragonal, and then to orthorhombic at around $\sim 230 \mathrm{~K}, \sim 160 \mathrm{~K}$ and $\sim 150 \mathrm{~K}$, respectively 10. Inorganic $\mathrm{CsPbBr}_{3}$ is cubic, (above $403 \mathrm{~K}$ ), tetragonal $(361 \mathrm{~K}<\mathrm{T}<403 \mathrm{~K}$ ), and orthorhombic (below 361 K) 11 13. Hybrid LHPs, i. e., those with organic cation $A$ are generally extremely challenging due to the ease of beam damage under visible and ultraviolet light irradiation [14, which complicates not only their use as an optoelectronic material, but also their study by spectroscopic methods.

Because of the $\mathrm{Pb}$ content, the spin-orbit interaction influences the electronic structure. This affects the band gap size and leads to spin-orbit splittings [15. The spinorbit interaction can cause a Rashba effect if a structural inversion asymmetry occurs at the surface or interfaces or if a bulk inversion asymmetry is present [16, 17. The strength of the Rashba effect is usually given by the Rashba parameter $\alpha_{\mathrm{R}}$, which is the ratio of energy splitting $\Delta E$ to the momentum shift $\Delta k$ of the electronic band structure $\left(\alpha_{\mathrm{R}}=\Delta E /(2 \Delta k)\right)$. In LHPs, ferroelectricity can break the inversion symmetry and cause a bulk Rashba splitting [18, also when $\mathrm{Pb}$ is replaced by $\mathrm{Sn}$ [19]. This prediction, originally obtained by tight binding calculations [18] has been confirmed in ab initio calculations using the GW approach [20, 21].

It has been suggested that appropriate spin textures caused by the Rashba effect can lead to a mismatch of spin and momentum in the recombination transitions
[22]. Calculations of the lifetime suggest that such suppression of the recombination can explain the observed long carrier lifetimes 22. It shall be noted that the Rashba effect can either slow down the transition due to the spin mismatch 22 or due to the mere resulting indirect band gap character 23, 24 which by itself would lead to a 350 -fold decelerated transition 24. I. e., already the difference in Rashba splittings in conduction band and valence band would turn halide perovskites into indirect band gap semiconductors 25]. This does not require a static Rashba effect since even in centrosymmetric structures a local Rashba effect can occur which fluctuates with the MA cation dynamics 25. Depending on structure and distortions, dynamical Rashba effects with parameters $\alpha_{\mathrm{R}}$ from 1.12 to $4.82 \mathrm{eV} \AA$ for the valence band and from 2.19 to $10.36 \mathrm{eV} \AA$ for the conduction band of $\mathrm{MAPbI}_{3}$ were calculated 25. These are very large values partly exceeding the largest effects measured in solids so far [16, 17. In most predictions the Rashba splitting of the conduction band is larger 25. or equal to that of the valence band [26. This changes, however, when surface Rashba effects are considered. A static surface Rashba effect has been predicted for the valence band of $\mathrm{MAPbI}_{3}$ that amounts to $\sim 2 \mathrm{eVA}$ with negligible effect in the conduction band [27].

Early on it has been noticed that in hybrid perovskites, the $\mathrm{Pb} X_{3}^{-}$unit determines the electronic and phononic properties 22]. Electronically, since there are no electronic states of the organic molecule in the vicinity of the band gap. And structurally, since the deformation of the $\mathrm{Pb} X_{3}^{-}$unit is well understood as the result of the influence of the organic molecule and has been confirmed as the source of the predicted Rashba splitting [22]. In line with this insight, phonon instabilities were found to be very similar in $\mathrm{CsPbI}_{3}$ and $\mathrm{MAPbI}_{3}$ [28] which is interesting since hybrid and inorganic perovskites also have comparable local polar fluctuations [29. By molecular dynamics simulations, two studies found a similar dynamical Rashba effect for $\mathrm{CsPbI}_{3}$ as in $\mathrm{MAPbI}_{3}$ [30, 31. For $\mathrm{CsPbI}_{3}$ in the static minimum, a Rashba parameter of $2.01 \mathrm{eVA}$ for the valence band and $2.75 \mathrm{eVA}$ for the 
conduction band has been found [31. Under external electric fields, $\mathrm{CsPbBr}_{3}$ can show a static Rashba effect which occurs only in the valence band [26. $\mathrm{A} \mathrm{CsPbBr}_{3}$ bilayer yields a Rashba parameter of $0.88 \mathrm{eV} \AA$ for 1.35 $\mathrm{V} / \mathrm{nm}$ field strength [26].

These predictions of Rashba effects call for experimental verification, especially since the magnitude of the Rashba splitting is a function of the polar distortion [22] and the splitting itself is not sufficient since many Rashba effects with untypical spin textures that do not impede the recombination can occur depending on the details of the distortions 32 .

Ferroelectricity has been measured for tetragonal $\mathrm{MAPbI}_{3}[33$. On the other hand, it has been argued that tetragonal $\mathrm{MAPbI}_{3}$ is centrosymmetric with $I 4 / \mathrm{mcm}$ space group which would exclude a bulk Rashba effect 34. However, single-crystal x-ray diffraction experiments show that tetragonal $\mathrm{MAPbI}_{3}$ belongs to the polar space group $I_{4} \mathrm{~cm}$, a subgroup of $14 / \mathrm{mcm}$ [35].

Angle-resolved photoelectron spectroscopy (ARPES) is the method of choice to investigate Rashba effects in the valence band [16, 17. In the case of $\mathrm{MAPbBr}_{3}$ [36, 37] and $\mathrm{MAPbI}_{3}$ [37, band dispersions could be extracted from the raw data. Niesner et al. searched for a possible Rashba splitting in $\mathrm{MAPbBr}_{3}$ by ARPES with and without circular dichroism at $21.2 \mathrm{eV}$ and $6.2 \mathrm{eV}$ photon energy [38. More specifically, the valence band maximum (VBM) was probed at the $\overline{\mathrm{M}}$ point at 21.2 $\mathrm{eV}$ where $E(k)$ dispersions were obtained by a leadingedge analysis followed by symmetrization. For both the low-temperature orthorhombic phase and the roomtemperature cubic phase, a local minimum of $0.16 \mathrm{eV}$ appeared in the $E(k)$ dispersions surrounded by a ring shaped maximum. Rashba parameters of $7 \pm 1 \mathrm{eV} \AA$ for the low-temperature orthorhombic phase and $11 \pm 4 \mathrm{eV}$ $\AA$ for the room-temperature cubic phase were obtained, exceeding the largest Rashba effects measured for ferroelectric materials [39, 40]. In a laser ARPES experiment, the Rashba splitting was reported at the $\bar{\Gamma}$ point $\left(k_{\|}=0\right)$, which contains a replica of the band at the VBM for the orthorhombic phase.

For a 2D hybrid perovskite, optical spectroscopies deliver an exciton splitting compatible with a Rashba parameter of $1.6 \mathrm{eV} \AA$ of the conduction band [41]. Magnetooptical measurements of $\mathrm{CsPbBr}_{3}$ nanocrystals were well described by Rashba parameters of $0.2 \mathrm{eV} \AA$ for the conduction band and $0.05 \mathrm{eVA}$ for the valence band [42]. From bulk-sensitive femtosecond absorption and photoluminescence at room temperature, it was concluded that the Rashba splitting observed in ARPES [38, can only occur at low temperature [43]. From second harmonic generation data, the Rashba effect in $\mathrm{MAPbI}_{3}$ was estimated to be only $1.3 \cdot 10^{-3} \mathrm{eV} \AA$ 44. From comparison to bulk-sensitive second-harmonic-generation experiments and calculations, Frohna et al. 34 conclude that Niesner et al. 38 accurately measured the surface
Rashba effect. Most recently, spin-polarized scanning tunneling spectroscopy was used to claim a measurable Rashba splitting at the surface of $\mathrm{MAPbI}_{3}$ [5].

In the present work, we have conducted ARPES from $\mathrm{MAPbBr}_{3}$ to verify the Rashba splitting of the size reported by Niesner et al. which is widely accepted [2, 8, 9, 26, 34, 41,43, 45. We obtained detailed dispersions of the valence band which are incompatible with the reported Rashba splittings. For $\mathrm{CsPbBr}_{3}$, ARPES does not give experimental evidence for a Rashba splitting [46]. $\mathrm{CsPbBr}_{3}$ has a higher chemical stability and allows also spin-resolved measurements. By this, we can exclude a static bulk or surface Rashba effect of $\sim 7.2 \cdot 10^{-2} \mathrm{eV} \AA$ or higher.

The hybrid $\mathrm{MAPbBr}_{3}$ and the $\mathrm{CsPBr}_{3}$ single crystals were grown using the inverse temperature [47] and the antisolvent vapor-assisted crystallization methods [48, respectively. We have doped $\mathrm{MAPbBr}_{3}$ crystals by $\mathrm{Mn}^{2+}$ in order to increase their chemical stability as suggested before for some LHPs [49, 50. ARPES and spin-resolved ARPES measurements were perfomed at the UE112PGM2a and U125/2-PGM RGBL Undulator beamlines of BESSY II. The angular and energy resolutions of the ARPES experiments were $0.1^{\circ}$ and $20 \mathrm{meV}$, respectively. Spin-resolved ARPES spectra were obtained via a Motttype spin detector [51]. Resolutions for spin-resolved ARPES were $1.5^{\circ}$ (angular) and $90 \mathrm{meV}$ (energy). The crystals were cleaved in situ along the [100] direction and the base pressure of the experimental setups was better than $1 \times 10^{-10}$ mbar (for more details, please see Supplemental Material [52.)

Figure 1(a) shows the bulk and surface Brillouin zones of the cubic perovskite lattice, and Fig. 1(b) displays the cubic crystal structure. The VBM is situated at the R-point which we access with angle scans where the $2 \mathrm{D}$ electron wave vector $\mathbf{k}_{\| y}$ is varied along $\bar{\Gamma}-\overline{\mathrm{M}}$ [i.e., within the yellow plane in Fig. 1(a)]. In previous experiments, it has been argued that the R-point of $\mathrm{MAPbBr}_{3}$ is reached at $21.2 \mathrm{eV}$ photon energy [37, 38. In Fig. 1(e) we show data measured at almost the same energy of $22 \mathrm{eV}$, where a small dispersive feature appears at lowest binding energies. This feature is a candidate for the VBM of $\mathrm{MAPbBr}_{3}$ and is displayed also in Fig. 1(f) as first derivative of photoemission intensity versus energy. The dispersion at $22 \mathrm{eV}$ does not indicate a Rashba splitting, in particular one does not see the $0.16 \mathrm{eV}$ dip at the top of the dispersion [38. Also our constant energy surfaces at the band maximum in Figs. 1(c) and 1(d) do not show the ring shape reported previously. We plotted the extracted data from [38 in Figs. 1(e) and 1(g) (yellow line) and find no agreement with our measurements. Besides the missing dip, our dispersion appears more pronounced than the one observed in the previous experiment 38 .

It is difficult to verify whether $22 \mathrm{eV}$ probes states near the R-point because the samples do not allow ex- 

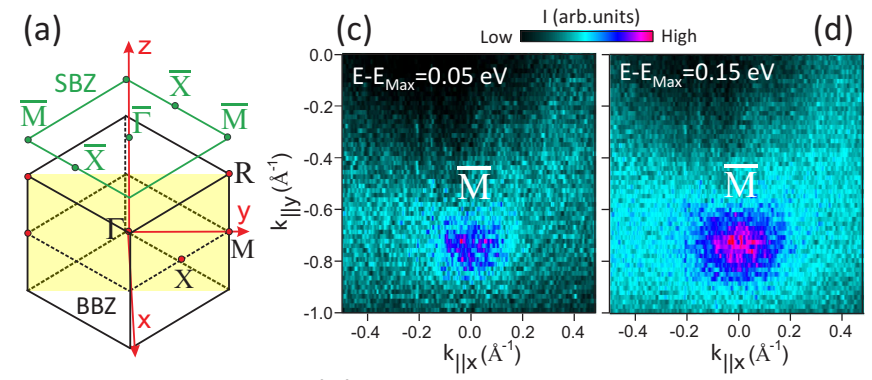

(b)
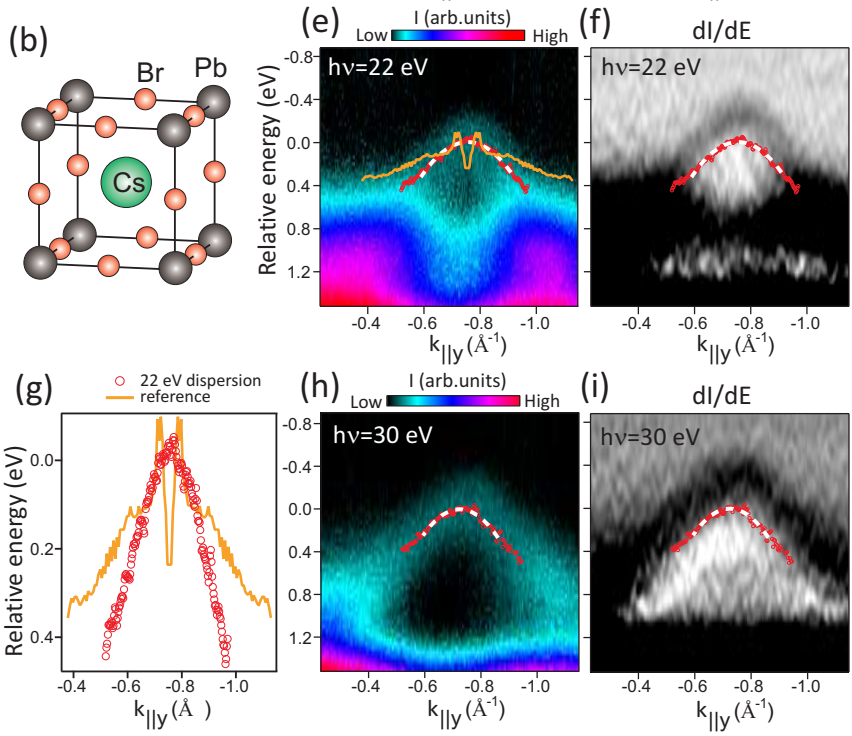

FIG. 1. (Color online) (a) Sketch of the surface (green) and bulk (black) cubic Brillouin zones. The yellow plane indicates the electron detection plane, which spans along the $\bar{\Gamma}-\overline{\mathrm{M}}$ direction. (b) Primitive unit cell of cubic perovskite. (c),(d) Constant-energy surfaces of hybrid $\mathrm{MAPbBr}_{3}$ measured around the $\overline{\mathrm{M}}$-point and at a relative energy with respect to the band maximum $\left(E-E_{\mathrm{Max}}\right)$ of (c) $0.05 \mathrm{eV}$ and (d) $0.15 \mathrm{eV}$. (e) Corresponding energy-momentum dispersion at $\mathrm{h} \nu=22 \mathrm{eV}$ photon energy and (f) first derivative of the photoemission intensity $(\mathrm{dI} / \mathrm{dE})$. Red circles are fits to the data, and the white dashed line is a parabolic fit to the resulting peak positions. (e),(g) The band dispersion extracted from Ref. 38 is shown for comparison (yellow solid line). (h),(i) Similar results as in (e),(f) but obtained at a photon energy of $\mathrm{h} \nu=30 \mathrm{eV}$.

tended measurement times. Absolute binding energies of different measurements cannot be compared because of energy shifts due to charging because of synchrotron or visible light and/or degradation. We observe, however, that data at $30 \mathrm{eV}$ show a more pronounced dispersion than at $22 \mathrm{eV}$, see Figs. 1(h) and 1(i). Our analysis of the effective mass gives $0.32 \pm 0.02 m_{e}$ for $22 \mathrm{eV}$, but $0.26 \pm 0.02 m_{e}$ for $30 \mathrm{eV}$. Although this difference is rather small, it indicates that $30 \mathrm{eV}$ may be closer to the R-point and the VBM.

For this reason we tested whether the more pronounced dispersion at $30 \mathrm{eV}$ shows any sign of a Rashba splitting. Since the dip is also absent here, we investigated the be-



FIG. 2. (Color online) $\mathrm{MAPbBr}_{3}$. Comparison between the widths of the photoemission peaks (black dots) and the results of the simulated model taking into account different Rashba parameters (red and green solid lines). The experimental widths are extracted from fits to energy-distribution curves at $\mathrm{h} \nu=30 \mathrm{eV}$ photon energy, and the corresponding error bars are shown in gray color.

havior of the dispersion with $\mathbf{k}_{\|}$. We simulated a model based on the Rashba effect [53] using the reported parameters $\alpha_{\mathrm{R}}=11 \mathrm{eV} \AA$ and $2 k_{0}=0.086 \AA^{-1}$ [38] which imply a value of $m^{*}=0.03 m_{e}$. The Rashba splitting must appear as an increased peak width for unresolved splittings as a function of $\mathbf{k}_{\|}$. Unresolved splittings are in particular expected for a dynamic Rashba effect [25]. This width is plotted in Fig. 2 (red solid line) using the full width at half maximum (FWFM) at the top of the band as reference width for the model [52]. We see from Fig. 2 that the experimental width behaves very differently from the simulation and maximally supports a Rashba parameter smaller by one order of magnitude, as can be seen when comparing the experimental data and their error bars to a simulation where $\alpha_{\mathrm{R}}=1.4 \mathrm{eV} \AA$ (green solid line). This comparison indicates again that the giant Rashba effect does not exist in $\mathrm{MAPbBr}_{3}$.

In the following, we turn to $\mathrm{CsPbBr}_{3}$. This system is much more stable and allows extended measurements. We determined by photon energy dependent measurements that the R-point is reached at $29 \mathrm{eV}$. Figure 3 shows the VBM. In Fig. 3(c) we perform a similar analysis as in Fig. 2, now for the VBM of $\mathrm{CsPbBr}_{3}$. Here, we use the experimental effective mass and assume a giant $\alpha_{\mathrm{R}}$ of $11 \mathrm{eV} \AA$ for the model. We see that the experimental data are not compatible with a giant Rashba effect, at most with a Rashba parameter of $1.06 \mathrm{eVA}$.

Niesner et al. also reported a circular dichroism effect 

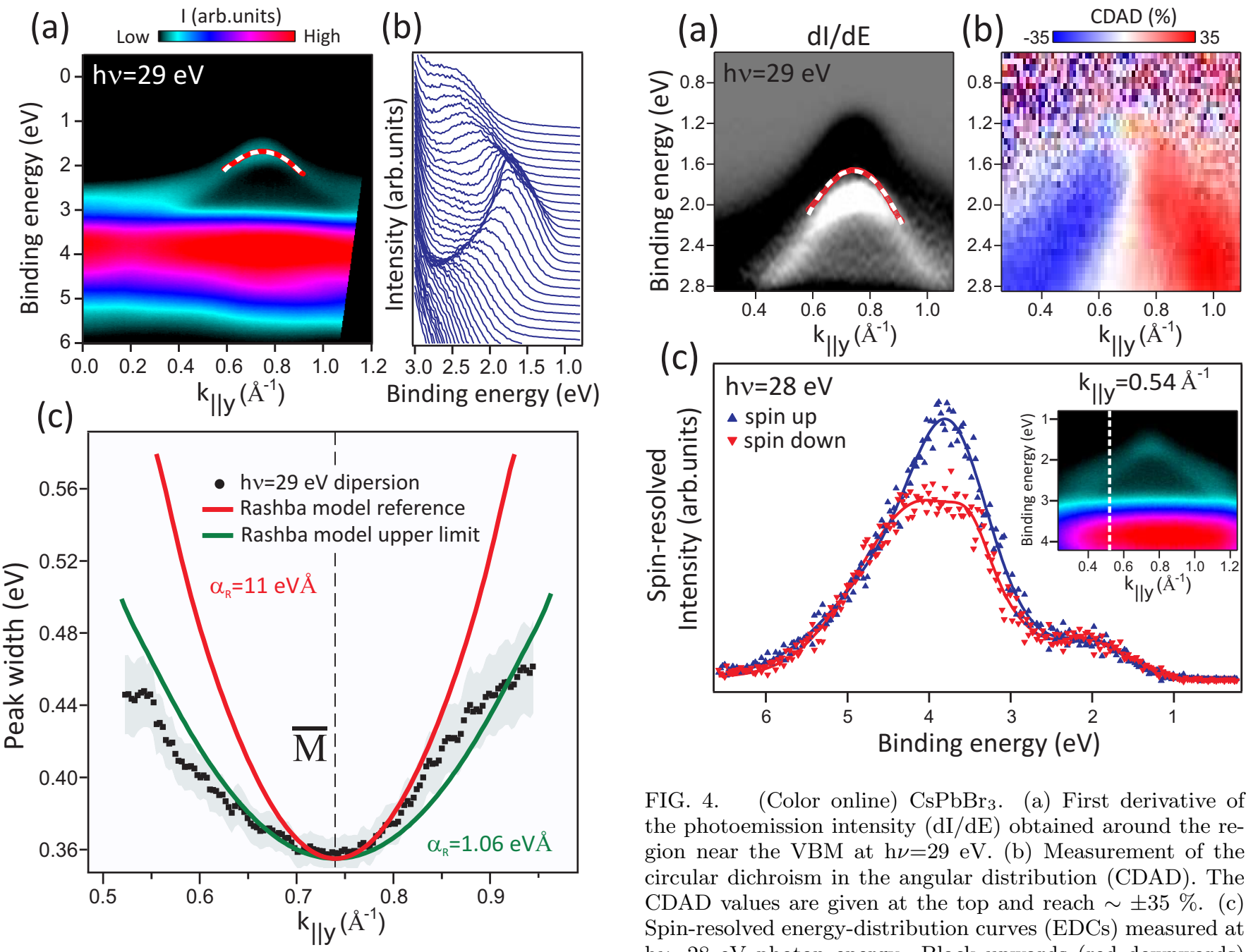

FIG. 4. (Color online) $\mathrm{CsPbBr}_{3}$. (a) First derivative of the photoemission intensity $(\mathrm{dI} / \mathrm{dE})$ obtained around the region near the VBM at $\mathrm{h} \nu=29 \mathrm{eV}$. (b) Measurement of the circular dichroism in the angular distribution (CDAD). The $\mathrm{CDAD}$ values are given at the top and reach $\sim \pm 35 \%$. (c) Spin-resolved energy-distribution curves (EDCs) measured at $\mathrm{h} \nu=28 \mathrm{eV}$ photon energy. Black upwards (red downwards) triangles show tangential spin-up (down) EDCs. The spin-up (down) direction lies in the surface plane and is perpendicular to the electron momentum. (For the out-of-plane component see Fig. S5 [52].) Solid lines are fits to the data. Inset: Corresponding energy-momentum dispersion at $\mathrm{h} \nu=28 \mathrm{eV}$. The vertical white dashed line indicates the momentum cut at which the spin-resolved spectra have been taken. parison between the width of the photoemission peaks dots) and the results of the simulated model using Rashba parameters of $\alpha_{\mathrm{R}}$ of $11 \mathrm{eV} \AA$ and $1.06 \mathrm{eVA}$ (red and green solid lines, respectively). The green curve represents an upper limit of the Rashba parameter as extracted from the model by taking into account the experimental error bars (gray color).

in $\mathrm{MAPbBr}_{3}$ as proof of the Rashba-type spin texture 38. With $6.2 \mathrm{eV}$ laser light, only the center of the surface Brillouin zone $\bar{\Gamma}$ can be reached. However, for the orthorhombic phase at low temperature, the bulk R-point is projected onto $\Gamma$ which could in principle be used to probe the VBM. We search for a circular dichroism at 29 $\mathrm{eV}$ and plot the intensities $\left(c_{+}-c_{-}\right) /\left(c_{+}+c_{-}\right)$in Fig. 4(b). They show a dichroism sign change at the VBM at the R-point. It has, however, previously been shown in detail for the Dirac cone surface state of $\mathrm{Bi}_{2} \mathrm{Te}_{3}$ that the circular dichroism is not related to the spin texture

and does not replace a spin-resolved ARPES experiment [54. In Fig. 4(c), we check with spin-resolved photoemission slightly away from the VBM at the R-point both the Rashba effect and the relevance of the circular dichroism for a spin splitting. We see at $28 \mathrm{eV}$ that the peak at $\sim 4 \mathrm{eV}$ binding energy leads to a strong spin polarization. This is consistent with the fact that the high spin-orbit interaction originates from the $\mathrm{Pb}$. This is also consistent with band structure calculations of $\mathrm{CsPbBr}_{3}$ which show that this $\mathrm{Pb} 6 s$ derived band splits strongly when spin-orbit coupling is taken into account [55. For the states near the VBM which mainly originate from $\mathrm{Br} 4 p$ orbitals 55, 56, we accordingly do not see such spin polarization. This shows that the circular dichroism is not related to a spin polarization. We also do not see a spin 
splitting that could be assigned to a Rashba effect. The spin splitting is smaller than $29 \pm 41 \mathrm{meV}$ corresponding to a static Rashba parameter $\alpha_{\mathrm{R}} \lesssim 7.2 \cdot 10^{-2} \mathrm{eVA}$. (Note that a dynamic Rashba effect would average over spin and not be detectable by spin resolution.) This is consistent with the values of $\alpha_{\mathrm{R}}$ in the range of $10^{-3}-10^{-2}$ $\mathrm{eV \AA}$ estimated from bulk-sensitive optical measurements of $\mathrm{CsPbBr}_{3}\left(5 \cdot 10^{-2} \mathrm{eV \AA}\right)$ [42 as well as of $\mathrm{MAPbI}_{3}$ $\left(1.3 \cdot 10^{-3} \mathrm{eV \AA}\right)$ 44.

In conclusion, we have investigated the presumed giant Rashba effect for $\mathrm{MAPbBr}_{3}$ and do not observe any static or dynamic effect in the reported order of magnitude, neither at 22 nor at $30 \mathrm{eV}$ photon energy. As for both hybrid and inorganic LHPs Rashba effects are predicted, we have also investigated the analogous but more stable inorganic compound $\mathrm{CsPbBr}_{3}$. Here, we can also exclude a large Rashba effect at the valence band maximum. Therefore, the high efficiency of hybrid and inorganic perovskite solar cells is most likely not caused by the spin texture or by the indirect band gap character of a valence-band Rashba effect in the bulk or at the surface.

\section{ACKNOWLEDGEMENTS}

We thank Eva Unger for helpful advice. Use of the Helmholtz Innovation Lab HySPRINT for sample preparation is gratefully acknowledged. We acknowledge financial support from the Impuls-und Vernetzungsfonds der Helmholtz-Gemeinschaft under grant No. HRSF-0067 (Helmholtz-Russia Joint Research Group).

[1] J. H. Noh, S. H. Im, J. H. Heo, T. N. Mandal, and S. I. Seok, Nano Lett. 13, 1764 (2013).

[2] D. P. McMeekin, G. Sadoughi, W. Rehman, G. E. Eperon, M. Saliba, M. T. Hörantner, A. Haghighirad, N. Sakai, L. Korte, B. Rech, et al., Science 351, 151 (2016)

[3] Y. Hou, E. Aydin, M. De Bastiani, C. Xiao, F. H. Isikgor, D.-J. Xue, B. Chen, H. Chen, B. Bahrami, A. H. Chowdhury, et al., Science 367, 1135 (2020).

[4] J. Xu, C. C. Boyd, J. Y. Zhengshan, A. F. Palmstrom, D. J. Witter, B. W. Larson, R. M. France, J. Werner, S. P. Harvey, E. J. Wolf, et al., Science 367, 1097 (2020)

[5] F. Sahli, J. Werner, B. A. Kamino, M. Bräuninger, R. Monnard, B. Paviet-Salomon, L. Barraud, L. Ding, J. J. D. Leon, D. Sacchetto, et al., Nat. Mater. 17, 820 (2018)

[6] E. Köhnen, M. Jošt, A. B. Morales-Vilches, P. Tockhorn, A. Al-Ashouri, B. Macco, L. Kegelmann, L. Korte, B. Rech, R. Schlatmann, et al., Sustain. Energy Fuels 3, 1995 (2019)

[7] Z. Yu, Sci. Rep. 6, 28576 (2016).
[8] J. Wang, C. Zhang, H. Liu, R. McLaughlin, Y. Zhai, S. R. Vardeny, X. Liu, S. McGill, D. Semenov, H. Guo, et al., Nat. Commun. 10, 1 (2019).

[9] V. V. Belykh, D. R. Yakovlev, M. M. Glazov, P. S. Grigoryev, M. Hussain, J. Rautert, D. N. Dirin, M. V. Kovalenko, and M. Bayer, Nat. Commun. 10, 1 (2019)

[10] C. Chen, X. Hu, W. Lu, S. Chang, L. Shi, L. Li, H. Zhong, and J.-B. Han, J. Phys. D: Appl. Phys. 51, 045105 (2018).

[11] M. Rodová, J. Brožek, K. Knížek, and K. Nitsch, J. Therm. Anal. Calorim. 71, 667 (2003).

[12] S. Hirotsu, J. Harada, M. Iizumi, and K. Gesi, J. Phys. Soc. Jpn. 37, 1393 (1974).

[13] M. Zhang, Z. Zheng, Q. Fu, Z. Chen, J. He, S. Zhang, L. Yan, Y. Hu, and W. Luo, CrystEngComm 19, 6797 (2017)

[14] N. Klein-Kedem, D. Cahen, and G. Hodes, Acc. Chem. Res. 49, 347 (2016).

[15] J. Even, L. Pedesseau, J.-M. Jancu, and C. Katan, J. Phys. Chem. Lett. 4, 2999 (2013).

[16] G. Bihlmayer, O. Rader, and R. Winkler, New J. Phys. 17, $050202(2015)$.

[17] A. Manchon, H. C. Koo, J. Nitta, S. Frolov, and R. Duine, Nat. Mater. 14, 871 (2015).

[18] M. Kim, J. Im, A. J. Freeman, J. Ihm, and H. Jin, PNAS 111, 6900 (2014)

[19] A. Stroppa, D. Di Sante, P. Barone, M. Bokdam, G. Kresse, C. Franchini, M.-H. Whangbo, and S. Picozzi, Nat. Commun. 5, 1 (2014)

[20] F. Brivio, K. T. Butler, A. Walsh, and M. Van Schilfgaarde, Phys. Rev. B 89, 155204 (2014)

[21] P. Umari, E. Mosconi, and F. De Angelis, Sci. Rep. 4, 4467 (2014)

[22] F. Zheng, L. Z. Tan, S. Liu, and A. M. Rappe, Nano Lett. 15, 7794 (2015).

[23] C. Motta, F. El-Mellouhi, S. Kais, N. Tabet, F. Alharbi, and S. Sanvito, Nat. Commun. 6, 7026 (2015)

[24] P. Azarhoosh, S. McKechnie, J. M. Frost, A. Walsh, and M. Van Schilfgaarde, APL Mater. 4, 091501 (2016)

[25] T. Etienne, E. Mosconi, and F. De Angelis, J. Phys. Chem. Lett. 7, 1638 (2016).

[26] X. Chen, L. Liu, and D. Shen, J. Phys.: Condens. Matter 30, 265501 (2018).

[27] E. Mosconi, T. Etienne, and F. De Angelis, J. Phys. Chem. Lett. 8, 2247 (2017).

[28] A. Marronnier, H. Lee, B. Geffroy, J. Even, Y. Bonnassieux, and G. Roma, J. Phys. Chem. Lett. 8, 2659 (2017)

[29] O. Yaffe, Y. Guo, L. Z. Tan, D. A. Egger, T. Hull, C. C. Stoumpos, F. Zheng, T. F. Heinz, L. Kronik, M. G. Kanatzidis, et al., Phys. Rev. Lett. 118, 136001 (2017).

[30] S. McKechnie, J. M. Frost, D. Pashov, P. Azarhoosh, A. Walsh, and M. Van Schilfgaarde, Phys. Rev. B 98, 085108 (2018)

[31] A. Marronnier, G. Roma, M. A. Carignano, Y. Bonnassieux, C. Katan, J. Even, E. Mosconi, and F. De Angelis, J. Phys. Chem. C 123, 291 (2018)

[32] M. Kepenekian, R. Robles, C. Katan, D. Sapori, L. Pedesseau, and J. Even, ACS Nano 9, 11557 (2015).

[33] Y. Rakita, O. Bar-Elli, E. Meirzadeh, H. Kaslasi, Y. Peleg, G. Hodes, I. Lubomirsky, D. Oron, D. Ehre, and D. Cahen, PNAS 114, E5504 (2017).

[34] K. Frohna, T. Deshpande, J. Harter, W. Peng, B. A. Barker, J. B. Neaton, S. G. Louie, O. M. Bakr, D. Hsieh, and M. Bernardi, Nat. Commun. 9, 1 (2018). 
[35] J. Breternitz, F. Lehmann, S. A. Barnett, H. Nowell, and S. Schorr, Angew. Chem. Int. Ed. 59, 424 (2020)

[36] C. Wang, B. R. Ecker, H. Wei, J. Huang, J.-Q. Meng, and Y. Gao, Phys. Chem. Chem. Phys. 19, 5361 (2017)

[37] F. Zu, P. Amsalem, D. A. Egger, R. Wang, C. M. Wolff, H. Fang, M. A. Loi, D. Neher, L. Kronik, S. Duhm, et al., J. Phys. Chem. Lett. 10, 601 (2019)

[38] D. Niesner, M. Wilhelm, I. Levchuk, A. Osvet, S. Shrestha, M. Batentschuk, C. Brabec, and T. Fauster, Phys. Rev. Lett. 117, 126401 (2016).

[39] M. Liebmann, C. Rinaldi, D. Di Sante, J. Kellner, C. Pauly, R. N. Wang, et al., Adv. Mater. 28, 560 (2016)

[40] J. Krempaský, H. Volfová, S. Muff, N. Pilet, G. Landolt, M. Radović, M. Shi, D. Kriegner, et al., Phys. Rev. B 94, 205111 (2016).

[41] Y. Zhai, S. Baniya, C. Zhang, J. Li, P. Haney, C.-X. Sheng, E. Ehrenfreund, and Z. V. Vardeny, Sci. Adv. 3, e1700704 (2017)

[42] M. Isarov, L. Z. Tan, M. I. Bodnarchuk, M. V. Kovalenko, A. M. Rappe, and E. Lifshitz, Nano Lett. 17, 5020 (2017)

[43] J. M. Richter, K. Chen, A. Sadhanala, J. Butkus, J. P. Rivett, R. H. Friend, B. Monserrat, J. M. Hodgkiss, and F. Deschler, Adv. Mater. 30, 1803379 (2018)

[44] Z.-G. Yu, J. Phys. Chem. C 122, 29607 (2018)

[45] A. Maiti, S. Khatun, and A. J. Pal, Nano Lett. 20, 292 (2020).

[46] M. Puppin, S. Polishchuk, N. Colonna, A. Crepaldi, D. N. Dirin, O. Nazarenko, R. De Gennaro, et al., Phys. Rev.
Lett. 124, $206402(2020)$

[47] M. I. Saidaminov, A. L. Abdelhady, B. Murali, E. Alarousu, V. M. Burlakov, W. Peng, I. Dursun, L. Wang, Y. He, G. Maculan, et al., Nat. Commun. 6, 1 (2015).

[48] Y. Rakita, N. Kedem, S. Gupta, A. Sadhanala, V. Kalchenko, M. L. Böhm, M. Kulbak, R. H. Friend, D. Cahen, and G. Hodes, Cryst. Growth Des. 16, 5717 (2016)

[49] S. Zou, Y. Liu, J. Li, C. Liu, R. Feng, F. Jiang, Y. Li, J. Song, H. Zeng, M. Hong, et al., J. Am. Chem. Soc. 139, $11443(2017)$

[50] Y. Wei, Z. Cheng, and J. Lin, Chem. Soc. Rev. 48, 310 (2019).

[51] G. Burnett, T. Monroe, and F. Dunning, Review of scientific instruments 65, 1893 (1994).

[52] See Supplemental Material at http://*** for more details on sample preparation, experimental methods, model simulation, and spin-resolved data.

[53] E. I. Rashba, Soviet Physics, Solid State 2, 1109 (1960).

[54] M. Scholz, J. Sánchez-Barriga, J. Braun, D. Marchenko, A. Varykhalov, M. Lindroos, Y. J. Wang, H. Lin, A. Bansil, J. Minár, et al., Phys. Rev. Lett. 110, 216801 (2013).

[55] E. Trushin, L. Fromm, and A. Görling, Phys. Rev. B 100, 075205 (2019)

[56] J. Yin, P. Maity, M. De Bastiani, I. Dursun, O. M. Bakr, J.-l. Brédas, and O. F. Mohammed, Sci. Adv. 3, e1701793 (2017). 